\title{
Home monitoring of breathing rate in people with chronic obstructive pulmonary disease: observational study of feasibility, acceptability, and change after exacerbation
}

\author{
This article was published in the following Dove Press journal: \\ International Journal of COPD \\ 20 April 2017 \\ Number of times this article has been viewed
}

\author{
Noah Rubio' \\ Richard A Parker ${ }^{2}$ \\ Ellen M Drost ${ }^{\prime}$ \\ Hilary Pinnock ${ }^{3,4}$ \\ Christopher J Weir ${ }^{2}$ \\ Janet Hanley ${ }^{5}$ \\ Leandro C Mantoani ${ }^{1}$ \\ William MacNee \\ Brian McKinstry 2,4 \\ Roberto A Rabinovich' \\ 'Edinburgh Lung and the Environment \\ Group Initiative (ELEGI) Colt \\ Laboratory, Centre for Inflammation \\ Research, ${ }^{2}$ Edinburgh Clinical Trials \\ Unit, Usher Institute of Population \\ Health Sciences and Informatics, The \\ University of Edinburgh, ${ }^{3}$ Allergy \\ and Respiratory Research Group, \\ Usher Institute of Population \\ Health Sciences and Informatics, \\ The University of Edinburgh, Medical \\ School, Teviot Place, ${ }^{4}$ EHealth group, \\ Usher Institute of Population Health \\ Sciences and Informatics, The \\ University of Edinburgh, ${ }^{5} \mathrm{School}$ of \\ Health and Social Care, Edinburgh \\ Napier University, Edinburgh, \\ Lothian, UK
}

Correspondence: Roberto A Rabinovich Edinburgh Lung and the Environment Group Initiative (ELEGI) Colt Laboratory, Centre for Inflammation Research, The Queen's Medical Research Institute, University of Edinburgh, 47 Little France Crescent, Edinburgh EHI6 4TJ, Scotland, UK

Tel +44 I3। 242920 I

Fax +44 I3I 2426582

Email roberto.rabinovich@ed.ac.uk
Abstract: Telehealth programs to promote early identification and timely self-management of acute exacerbations of chronic obstructive pulmonary diseases (AECOPDs) have yielded disappointing results, in part, because parameters monitored (symptoms, pulse oximetry, and spirometry) are weak predictors of exacerbations.

Purpose: Breathing rate (BR) rises during AECOPD and may be a promising predictor. Devices suitable for home use to measure BR have recently become available, but their accuracy, acceptability, and ability to detect changes in people with COPD is not known.

Patients and methods: We compared five BR monitors, which used different monitoring technologies, with a gold standard (Oxycon Mobile ${ }^{\circledR}$; CareFusion ${ }^{\circledR}$, a subsidiary of Becton Dickinson, San Diego, CA, USA). The monitors were validated in 21 stable COPD patients during a 57-min "activities of daily living protocol" in a laboratory setting. The two best performing monitors were then tested in a 14-day trial in a home setting in 23 stable COPD patients to determine patient acceptability and reliability of signal. Acceptability was explored in qualitative interviews. The better performing monitor was then given to 18 patients recruited during an AECOPD who wore the monitor to observe BR during the recovery phase of an AECOPD.

Results: While two monitors demonstrated acceptable accuracy compared with the gold standard, some participants found them intrusive particularly when ill with an exacerbation, limiting their potential utility in acute situations. A reduction in resting BR during the recovery from an AECOPD was observed in some, but not in all participants and there was considerable day-to-day individual variation.

Conclusion: Resting BR shows some promise in identifying exacerbations; however, further prospective study to assess this is required.

Keywords: COPD exacerbation, telemedicine, COPD management, heart rate

\section{Introduction}

Worldwide, 65 million people live with COPD, and this number is predicted to increase by one-third by $2030 .{ }^{1}$ Self-management, supporting early identification, and timely treatment of acute exacerbations of COPD (AECOPD) is promoted to reduce hospital admissions, improve time to recovery, slow disease deterioration, and reduce health care use $/$ cost. $^{2-8}$ As $>50 \%$ of the costs of COPD are due to hospital admissions, reducing these is a priority for health services. ${ }^{9}$

Globally, health care policies encourage telehealthcare-supported self-management of long-term conditions, but current telehealth systems are limited by what can 
be feasibly monitored in a domestic setting and in terms of cost-effectively preventing hospital admissions; results have been disappointing. ${ }^{10-15}$ This, in part, is due to the poor predictive ability of currently available physiological measurements and symptom scores. ${ }^{16}$ There is a need for convenient and reliable measures that predict AECOPD. Breathing rate (BR) increases early during AECOPD and is thus a potential target for telemonitoring for the early identification and treatment of AECOPD to prevent hospital admission. ${ }^{17}$

Historically, methods of measuring BR were intrusive and unsuitable for routine domiciliary use, but recently less obtrusive technologies have been developed, initially for use in sports and military environments. However, it is not clear if devices that work well in fit people will also be reliable in breathless people with COPD. Identification and validation of devices that can reliably and conveniently be used to monitor $\mathrm{BR}$ in patients with COPD is a first step in the assessment of BR as a potential early indicator of AECOPD.

This study was conducted in three phases. In phase I, we aimed to select and validate commercially available devices to determine if they could accurately monitor BR in people with a broad spectrum of COPD severity. In phase II, the two best performing monitors were used at home by patients and their acceptability and perceived utility assessed qualitatively. In phase III, we aimed to examine the ability of the best performing device to detect changes in BR as patients recovered from an acute exacerbation with the aim, that if it proved feasible to detect such changes, to carry out a future prospective study in a group of patients at risk of AECOPD to see if BR changes might be useful in identifying exacerbations at an early stage.

\section{Methods}

\section{Approvals}

The study was approved by the National Health Service (NHS) Health Research Authority South East Scotland Research Ethics Committee (13/SS/0114, 13/SS/0206, 14/SS/0043) and received governance approval from NHS Lothian.

\section{Patient recruitment}

We recruited patients with moderate-to-very severe COPD as defined in the Global Initiative for Chronic Obstructive Lung Disease (GOLD) guidelines. ${ }^{18}$ In phases I and II, participants were recruited from respiratory outpatient clinics. They had to be clinically stable and free of exacerbations for at least 6 weeks prior to recruitment. Patients were recruited for phase III during an acute exacerbation either as hospital inpatients or from the local community respiratory team at home. Patients were excluded if they had terminal prognosis, if they had a heart pacemaker or automatic defibrillator, or if they had evidence of skin irritation (several devices were applied to skin). Written informed consent was taken from all patients for this study.

\section{Study Design}

Phase I: validation of BR measurements in a laboratory setting

\section{Characterization of participants}

Participants undertook spirometry (Alpha Spirometer; Vitalograph, Buckingham, Buckinghamshire, UK) according to American Thoracic Society/European Respiratory Society standards before and after the administration of $2.5 \mathrm{mg}$ of nebulized salbutamol. ${ }^{19}$ As a measure of muscle function, muscle strength was assessed as the maximal isometric quadriceps voluntary contraction using a strain gauge dynamometer (Chatillon ${ }^{\circledR}$; K-MSC 500, Ametek, FL, USA). ${ }^{20,21}$

Participants wore four BR monitors simultaneously in contact with the skin while being video-monitored by a fifth. The monitors' technical characteristics and the pseudonyms used in this study are shown in Table 1. Briefly, these were a chest mounted electrode array (measuring impedance), a finger mounted photoplethysmography system (measuring beat-to-beat variation), a camera mounted distance photoplethysmography device (measuring chest wall movements and skin color change), an upper abdomen mounted triaxial accelerometer, and a chest worn pressure sensor pad on elastic band, which also included a triaxial accelerometer and electrocardiograph.

Patients were asked to perform a set of activities during a standardized protocol of activities over 57-min, chosen to be representative of daily life activities, such as sitting, standing, walking, lifting, and climbing stairs. The protocol combined activities of different intensities and resting periods to stimulate changes in BR (Table 2). The aim was to assess the accuracy of the monitors in measuring BR and in detecting changes of different magnitudes in BR.

The gold standard against which the five monitors were tested was a metabolic system (Oxycon Mobile ${ }^{\circledR}$; CareFusion $^{\circledR}$, a subsidiary of Becton Dickinson, San Diego, CA, USA) that the patients also wore together with an oxygen saturation ear probe and a Polar T31 (Polar Electro [UK] Ltd, Warwick, Warwickshire, UK) coded transmitter belt for heart rate (HR) monitoring. The Oxycon was attached to the upper chest with a harness that caused minimal discomfort due to its low weight (950 g). A facemask with a dead 
Table I Monitors' technical characteristics

\begin{tabular}{|c|c|c|c|c|}
\hline Monitor & Output & Size & Position & Technology \\
\hline Monitor I: Impedance & $\mathrm{ECG}, \mathrm{HR}, \mathrm{BR}$, and PAL & $7.5 \times 5.0 \times 2.0 \mathrm{~cm}$, weight $60 \mathrm{~g}$ & $\begin{array}{l}\text { Attached to the chest and upper } \\
\text { abdomen }\end{array}$ & $\begin{array}{l}\text { Electrode array } \\
\text { (impedance) }\end{array}$ \\
\hline Monitor 2: PPG & $\mathrm{BR}$ & $56 \times 36 \times 62 \mathrm{~mm}$, weight $54 \mathrm{~g}$ & $\begin{array}{l}\text { Worn on the wrist with a finger } \\
\text { probe }\end{array}$ & PPG \\
\hline Monitor 3: Camera & $B R$ and $H R$ & N/A & $\begin{array}{l}\text { Participant was videoed while in } \\
\text { sitting position }\end{array}$ & Distance PPG algorithm \\
\hline Monitor 4: Accel & $B R$ and $P A L$ & $4.5 \times 3.5 \times 1.3 \mathrm{~cm}$, weight $18 \mathrm{~g}$ & $\begin{array}{l}\text { Attached to the upper abdomen just } \\
\text { below the ribs and taped to the skin }\end{array}$ & Accelerometer \\
\hline Monitor 5: Chest-band & $\begin{array}{l}\text { HR, R-R Interval, BR, } \\
\text { ECG, posture, PAL } \\
\text { - Posture } \\
\text { - Activity level } \\
\text { - Peak Accel }\end{array}$ & $\begin{array}{l}2.8 \times 0.7 \mathrm{~cm} \text {, weight } 89 \mathrm{~g} \\
\text { (including the chest strap) }\end{array}$ & $\begin{array}{l}\text { Chest strap and an electronics } \\
\text { module that attaches to the strap }\end{array}$ & $\begin{array}{l}\text { Pressure sensor pad } \\
\text { and accelerometer }\end{array}$ \\
\hline
\end{tabular}

Abbreviations: BR, breathing rate; ECG, electrocardiogram; HR, heart rate; PAL, physical activity level; PPG, photoplethysmography; N/A, not applicable; R-R, distance between $2 \mathrm{R}$ peaks on ECG; Accel, accelerometer.

space $<30 \mathrm{~mL}$ (Hans Rudolph Inc, Kansas City MO, USA) was used. The location of the Oxycon device did not interfere with the functioning of the BR monitors. The Oxycon device was calibrated (a two-point gas calibration) prior to each test. As part of the calibration process, the internal clocks of all the BR monitors were synchronized with the Oxycon to ensure optimal alignment of the different measurements. The Oxycon continuously measured oxygen consumption $\left(\mathrm{VO}_{2}\right)$, carbon dioxide production $\left(\mathrm{VCO}_{2}\right), \mathrm{HR}, \mathrm{BR}$, and tidal volume. Breath-by-breath measurements were averaged over $1-$ min intervals.

Monitor 5 (Chest-band) also provided data on HR and physical activity level (PAL). Comparison of the HR provided

Table 2 Protocol followed to assess BR variations during the phase I of the study

\begin{tabular}{ll}
\hline Type of activity & Time (min) \\
\hline Lying & 4 \\
Sitting & 2 \\
Standing & $\mathrm{I}$ \\
Slow 6MWT & 6 \\
Sitting & 3 \\
Fast 6MWT & 6 \\
Sitting & 5 \\
Sweeping & 4 \\
Sitting & 2 \\
Lifting objects & 3 \\
Sitting & 2 \\
Standing and walking & $\mathrm{I}$ \\
Climbing stairs & $\mathrm{I}$ \\
Sitting & 5 \\
Standing and walking & $\mathrm{I}$ \\
Treadmill (flat walking) & 4 \\
Treadmill (4\% slope) & 4 \\
Standing and walking & $\mathrm{I}$ \\
Sitting & 2 \\
\hline
\end{tabular}

Abbreviations: BR, breathing rate; $6 \mathrm{MWT}, 6$ minutes walking test. by the Monitor 5 (Chest-band) with the HR provided by Oxycon was used to validate this parameter. To validate PAL provided by the Monitor 5 (Chest-band), Oxycon $\mathrm{VO}_{2}$ values were divided by participants' body weight and converted to metabolic equivalents of task (METs). ${ }^{22}$ These METs energy expenditure estimates were used as a measure of energy expenditure to validate PAL provided by Monitor 5 (Chestband), and to identify the BR data recorded when patients were at rest (see the file online supplement in Supplementary material for further detail).

In addition, during this phase, patients were asked which monitor they preferred. Data were downloaded from all the devices to a personal computer for analysis.

\section{Analysis}

In phase I, minute-by-minute data from all devices were compiled for each patient in one database, and synchronization was verified by inspection of the curves of the $\mathrm{VO}_{2}$ in relation to the activities performed to ensure the best fit between the monitors on a patient-by-patient basis. Participant differences between the BR measured with each trial device and the Oxycon device were plotted against the average of the values from each device and the Oxycon device (BlandAltman plots). ${ }^{23}$ Linear mixed effects models were used to compute the upper and lower $95 \%$ limits of agreement (LoA) of the differences; taking into account multiple sources of variation due to patients and activities by modeling these as random and fixed effects respectively. Ninety-five percent confidence intervals were computed around the limits using a bootstrap method.

The two monitors that proved to be the most accurate (lowest mean bias and narrowest LoA) in measuring BR were selected for further assessment. 
Phase II: performance at home and qualitative assessment of acceptability

In phase II, 23 stable patients were recruited and asked to wear the two BR monitors simultaneously during waking hours over 14 consecutive days. Patients were asked to take two 30-min resting periods every day (circa 12:00 h and 19:00 h) during which time they wore a pulse oximeter and were asked to complete the Borg and modified Medical Research Council dyspnea scales. Patients recorded these data and rest times in a study diary. After 14 days of monitoring, data were downloaded to a personal computer.

\section{Qualitative assessment}

A semi-structured interview was arranged with each patient in their home after the monitoring period to explore their perceptions of using the technology. The interviews were based on an interview guide, which was revised iteratively as the study progressed (see the file online supplement in Supplementary material for the topic guide, section 2.6). ${ }^{24}$ The interviews were conducted by a researcher not involved with the quantitative monitoring or analysis to reduce the possibility of bias. Interviews were audio-recorded, fully transcribed, inductively coded, and analyzed thematically to explore the patient's experience of wearing the BR monitors.

\section{Quantitative analysis}

Overall BR data were summarized descriptively (mean, median, upper and lower quartile, and maximum and minimum). Using daily resting BR data, we established an "at-rest" reference range for the COPD population. Normal linear mixed models were used to determine the between-patient and day-to-day within-patient variation in breathing measurements for each device, including the patient as a random effect.

To determine the measurement period of time necessary to provide a stable indication of resting BR, we calculated the duration of monitoring required to achieve a $95 \%$ confidence interval half-width of two breaths per minute (bpm) for the upper and lower limits of the reference range.

\section{Phase III: ability to capture changes in BR during recovery from an AECOPD}

The monitor that performed best in terms of accuracy and patient preference (Chest-band) was selected for phase III. Patients were recruited during hospital admission with AECOPD or by the community respiratory team during an AECOPD managed at home. Each patient was asked to wear the device from the day of recruitment while in the ward (severe AECOPD) or their homes (moderate/severe
AECOPD), during waking hours, over a period between 3 and 6 weeks. On completion, data from the monitor were downloaded to a personal computer.

The evolution of BR data up to 41 days following AECOPD was summarized descriptively and graphically for each patient and overall. As in phase II, normal linear mixed models were used to determine the between-patient and dayto-day within-patient variation in "at-rest" BR measurements, with the addition of a fixed effect for number of days postexacerbation to adjust for changes in BR over time. Very low BRs of below $10 \mathrm{bpm}$ or very high values of 40 or above were regarded as implausible and excluded from the analysis.

Statistical analyses were performed using R software (version 3.2.3) and SAS software (version 21). ${ }^{25,26}$ Figure construction was performed with GraphPad Prism Version 4.0 and R software.

\section{Availability of data and materials}

Further information is included in the file online supplement in Supplementary material and is available on request from the corresponding author.

\section{Results}

\section{Phase I.Validation of BR measurements in a laboratory setting}

The characteristics of the phase I participants are shown in Table 3 and the Bland and Altman plots are shown in Figure 1.

Table 3 Patients characteristics

\begin{tabular}{|c|c|c|c|}
\hline & $\begin{array}{l}\text { COPD } \\
\text { patients } \\
\text { phase I }\end{array}$ & $\begin{array}{l}\text { COPD } \\
\text { patients } \\
\text { phase II }\end{array}$ & $\begin{array}{l}\text { COPD } \\
\text { patients } \\
\text { phase III }\end{array}$ \\
\hline $\mathrm{n}$ & 21 & 23 & 18 \\
\hline Men (\%) & $13(62)$ & $13(65)$ & $9(69)$ \\
\hline Age (years) & 68.7, SD 7.9 & $68.8, S D 7.6$ & 71.5, SD 10.4 \\
\hline Height (cm) & I66.3, SD 8.4 & 165.4, SD II.9 & 165.5, SD 9.9 \\
\hline Weight (kg) & 7I.I, SD I7 & 76.2, SD 22.I & 75.5, SD I5.8 \\
\hline BMI $\left(\mathrm{kg} / \mathrm{m}^{2}\right)$ & 25.6, SD 5.7 & 28.6, SD 9.5 & 27.5, SD 5.1 \\
\hline Pack/year & 37.0, SD I8.0 & 46.5, SD 19.6 & 53, SD 20.7 \\
\hline $\mathrm{mMRC}$ & & I.I5, SD I & 3.3, SD 0.7 \\
\hline $\mathrm{FEV}_{1}(\mathrm{~L})$ & I.4, SD 0.5 & I.24, SD 0.5 & I.2, SD 0.5 \\
\hline $\mathrm{FEV}_{1}$ (\% pred) & 47 (59\%) & $42(53 \%)$ & 42 (53\%) \\
\hline $\mathrm{FVC}(\mathrm{L})$ & 3.0, SD I.0 & 2.6, SD 0.7 & 2.7, SD I.I \\
\hline FVC (\% pred) & 81 (101\%) & $7 \mid$ (88\%) & 75 (94\%) \\
\hline $\mathrm{FEV}_{1} / \mathrm{FVC}$ & 0.5, SD 0.47 & 0.48, SD 0.45 & 0.44, SD 0.45 \\
\hline $\mathrm{SpO}_{2}(\%)$ & & 92.0, SD 3.7 & 91.I, SD 4.6 \\
\hline 6MWD (m) & 432.8, SD I 24.7 & & \\
\hline QMVC (kg) & 3I.6, SD 9.2 & & \\
\hline PAL (steps) & 4,368, SD 2,270 & & \\
\hline
\end{tabular}

Abbreviations: 6MWD, 6 minutes walking distance; BMI, body mass index; COPD, chronic obstructive pulmonary disease; FEV , forced expiratory volume in the first second; \% pred, percent of predicted; FVC, forced vital capacity; mMRC, modified Medical Research Council dyspnea score; pack/year, cumulative history of smoking; $\mathrm{PAL}$, physical activity level; QMVC, quadriceps maximal voluntary contraction; $\mathrm{SpO}_{2}$, oxygen saturation; SD, standard deviation. 

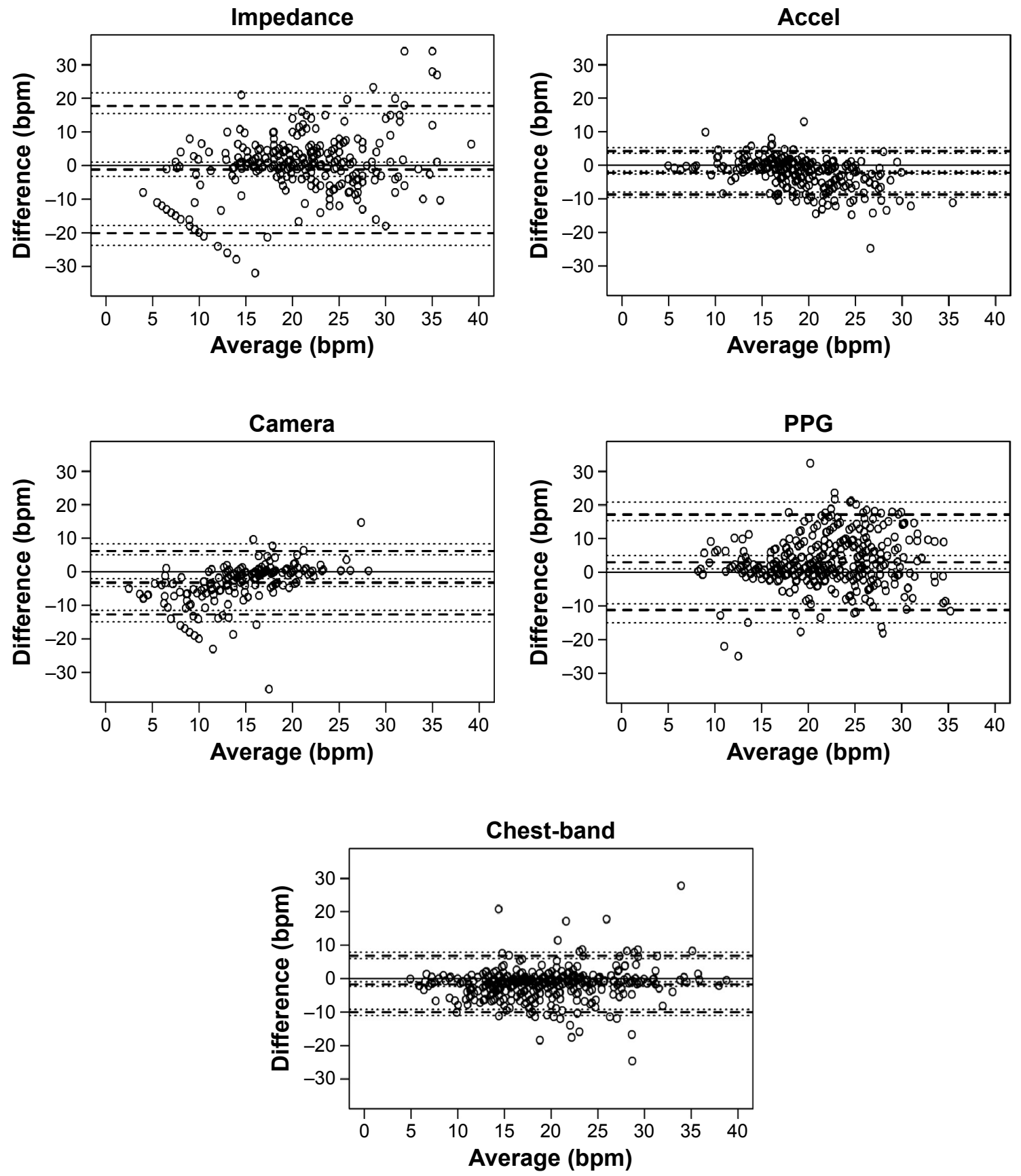

Figure I Validation of BR in a laboratory setting for five monitors.

Notes: Bland and Altman plots between Oxicon ${ }^{\circledR}$ BR output and different monitors BR outputs. Solid lines represent the bias, and dotted lines represent $95 \%$ limits of prediction. Abbreviations: Accel, accelerometer; bpm, breath per minute; BR, breathing rate; PPG, photoplethysmography.

Monitor 4 (accelerometer [Accel]; bias $=-2.18, \mathrm{LoA}=-8.63$ to 4.27) and Monitor 5 (Chest-band; bias $=-1.60$, LoA $=-9.99$ to 6.80 ) showed lower bias and a narrower LoA than Monitor 1 (impedance; bias $=-1.18, \mathrm{LoA}=-20.07$ to 17.72 ), Monitor 2 (photoplethysmography [PPG]; bias=3.01, LoA $=-11.17$ to 17.19 ) and Monitor 3 (Camera; bias $=-3.21, \mathrm{LoA}=-12.71$ to 6.30 ). Bias and LoA are shown in Table 4.

The ability to capture changes in BR was tested using BR data obtained at rest (sitting immediately before the activity; mean 15.6, SD $1.4 \mathrm{bpm}$ ) and during activity (fast 6 minute walking test [6MWT], slow 6MWT, sweeping, and lifting objects; Figure 2; mean 21.0, SD 0.2 bpm). Monitor 5 (Chest-band) showed the best concordance with the data measured by the gold standard and the greatest sensitivity to changes in activity. The PAL and HR provided by Monitor 5 were also validated in phase I (see the file online supplement in Figures S1 and $\underline{\mathrm{S} 2}$ ). The two devices selected to proceed to phase II were Monitor 4 (Accel) and Monitor 5 (Chest-band). 
Table 4 Bias and limits of agreement of the five monitors

\begin{tabular}{lll}
\hline Monitors & Bias & Limit of agreement \\
\hline Monitor I (Impedance) & -1.18 & -20.07 to 17.72 \\
Monitor 2 (PPG) & 3.01 & -11.17 to 17.19 \\
Monitor 3 (Camera) & -3.21 & -12.71 to 6.30 \\
Monitor 4 (Accel) & -2.18 & -8.63 to 4.27 \\
Monitor 5 (Chest-band) & -1.60 & -9.99 to 6.80 \\
\hline
\end{tabular}

Abbreviation: PPG, photoplethysmography.

\section{Phase II. Performance at home and}

\section{qualitative assessment of acceptability}

There were design and acceptability issues with both monitors. The adhesive patch used with Monitor 4 (Accel) caused skin problems in some people and it did not always keep the device in place. Most patients were confident placing Monitor 5 (Chest-band) though some had difficulties in fitting it comfortably and a few, whose body shape was outside the norm, could not wear the device in the recommended position and some women had difficulty fitting it under their bra. Some patients reported difficulty in removing the Monitor 5 (Chestband) device from the chest strap to charge the monitor.

The general preference for patients was for the Monitor 5 (Chest-band). The main reasons for this were because of problems with the adhesive pouch used to keep Monitor 4 (Accel) in place. The interview schedule included questions about the utility of the devices and the concept of BR monitoring. As neither of the monitors provided any direct feedback to the patients (data had to be downloaded and processed), patients found it hard to speculate about self- or nurse-led monitoring of their breathing. However, most agreed in principle that this would be desirable (see the file online supplement in Supplementary material for the interview guide, section 2.1).

Out of a possible total of 644 readings, 237 (37\%) valid resting BR measurements were recorded using Monitor 4 (Accel), compared to 351 (55\%) for Monitor 5 (Chest-band). Measured bpm ranged from 9.0 to 30.2 using Monitor 4 (Accel) and from 9.5 to 45.7 using Monitor 5 (Chest-band) (see the file online supplement in Supplementary material section 2.2 for data completeness). The overall mean BR was 21.5 (SD 3.8) for Monitor 4 (Accel) compared to 18.8 (SD 4.9) for Monitor 5 (Chest-band). The within-patient standard deviation of BR was estimated at 2.92 for Monitor 4 (Accel) compared to 3.36 for Monitor 5 (Chest-band) (see the file online supplement in Supplementary material section 2.3
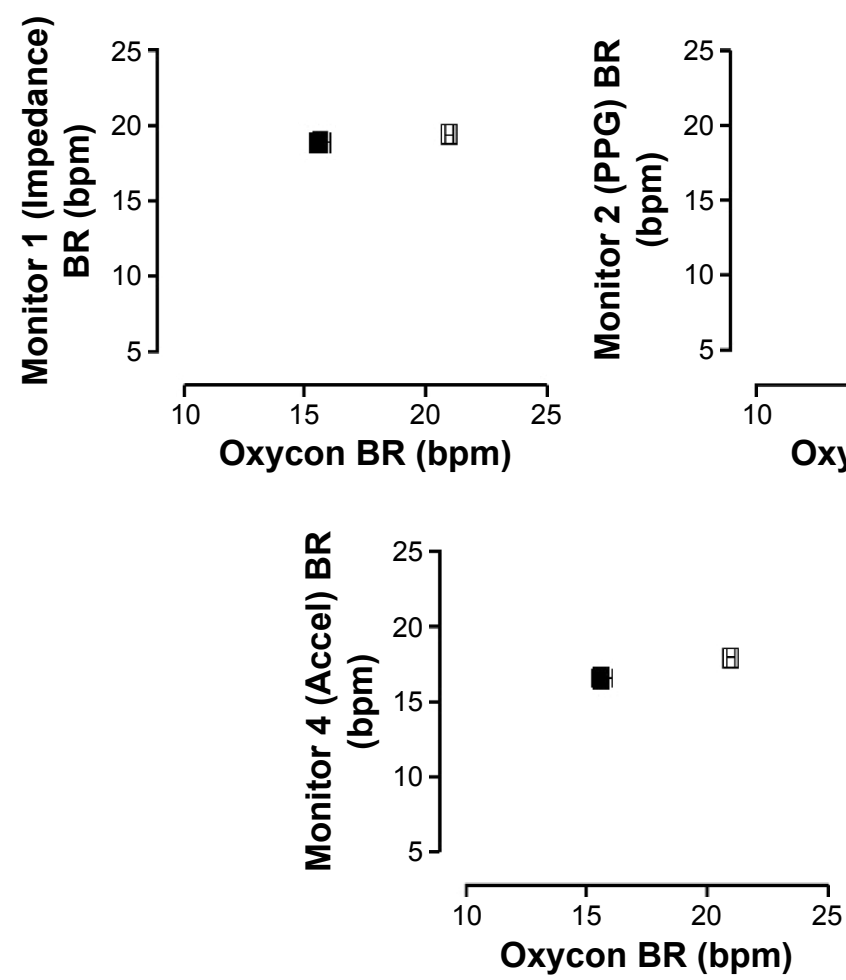
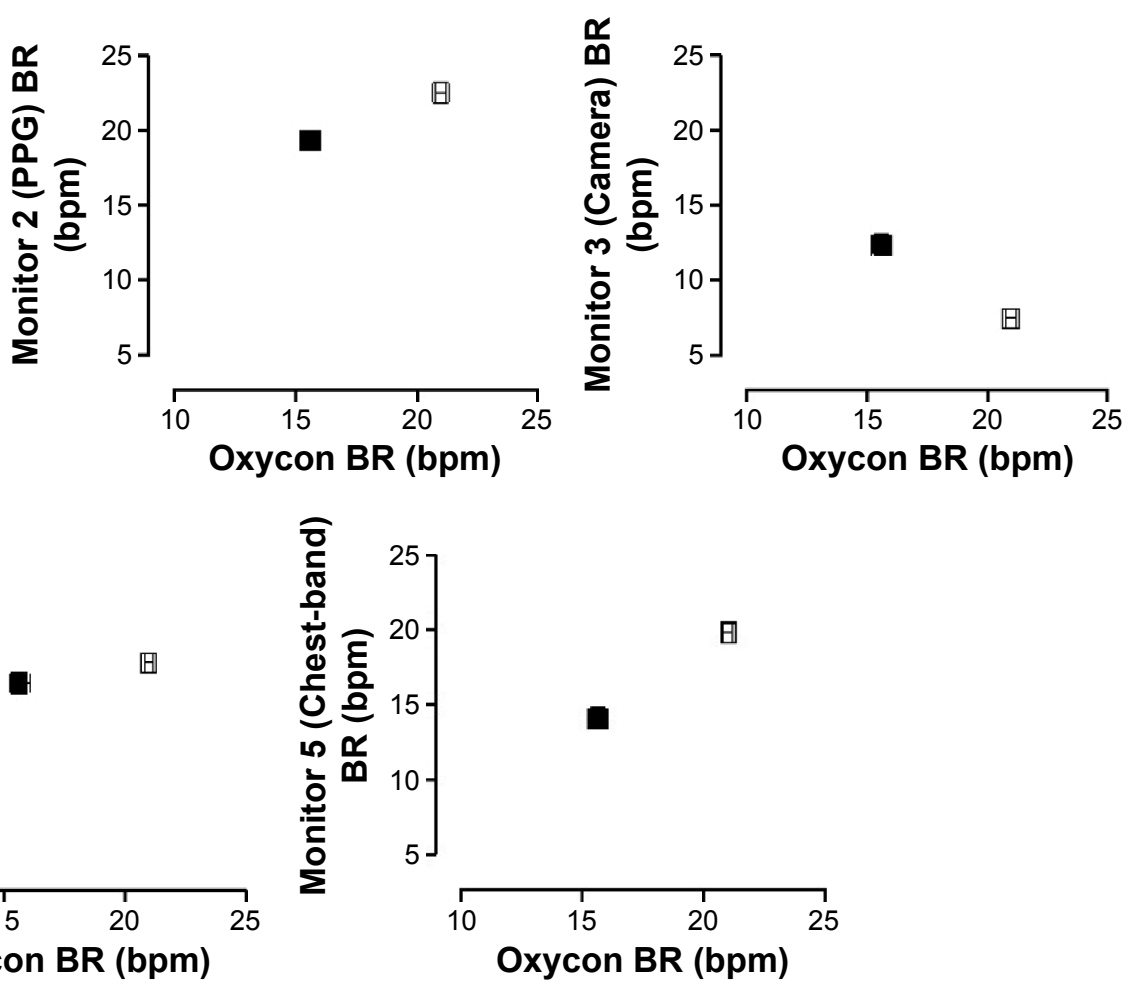

Sitting $\square$ Activity

Figure 2 Plots of the mean Oxicon ${ }^{\circledR}$ BR output against the mean output for the other monitors when comparing resting (sitting) with combined activities (fast 6MWT, slow 6MWT, sweeping, and lifting).

Abbreviations: 6MWT, 6 minute walking test; bpm, breath per minute; PPG photoplethysmography; BR, breathing rate. 
for respiratory rate statistics; see the file supplement tables in Table S1 for within-patient variances of BR). At least 25 days of measurement using Monitor 4 (Accel) would be needed for an at rest $\mathrm{BR}$ reference range to be estimated with sufficient precision ( $\pm 2 \mathrm{bmp})$. For Monitor 5 (Chest-band), $\sim 33$ days of measurement would be required. (See the file supplement tables in Table S2 for random effects modeling of the "at-rest" respiratory rate data for Monitors 4 and 5; see the file supplement tables in Table S3a and $\underline{b}$ [Monitor 5] and [Monitor 4] for individual patient 95\% reference ranges; see section 2.6 and the file supplement tables in Table S4 in the Supplementary material for results from normal linear mixed models for the association between respiratory rate and other variables.)

Monitor 5 (Chest-band) was selected for further testing in phase III on the basis of its performance in collecting usable data and patients' preferences.

\section{Phase III. Ability to capture changes during recovery from an AECOPD}

Monitor 5 (Chest-band) was the only monitor used in this phase. A total of 19 patients were recruited into the study, one of whom withdrew consent before any monitoring was done. Therefore, there were 18 patients with at least one valid observation recorded. One patient died during follow-up. Five other patients withdrew from the study early. The main reason for nonparticipation and withdrawal was that they felt too ill to participate or to continue. Two patients ( 3 and 4 ) had a second AECOPD event during their monitoring period, thus providing data from two exacerbations in these patients.

Monitor 5 (Chest-band) also measured physical activity data (see the file online supplement in Supplementary material). We were thus able to exclude those BR observations where the patient was evidently not at rest, instead of relying on a patient report as we had planned. Therefore, for the analysis in this phase, we only used BR data for which we were confident that the patient was "at rest".

In total, 109,686 BR observations were recorded as valid. The average number of observations per patient was 6,094 (range 135-28,836; see the file online supplement in Supplementary material section 3.2 for descriptive statistics of resting respiratory rate).

The within-patient standard deviation was estimated to be 3.99 and between-patient standard deviation 2.98 (see the file online supplement in Supplementary material section 3.3 for variation in resting respiratory rate measurements). The plot of the mean BR from all patients suggests a gradual decrease in resting $\mathrm{BR}$ over time following an

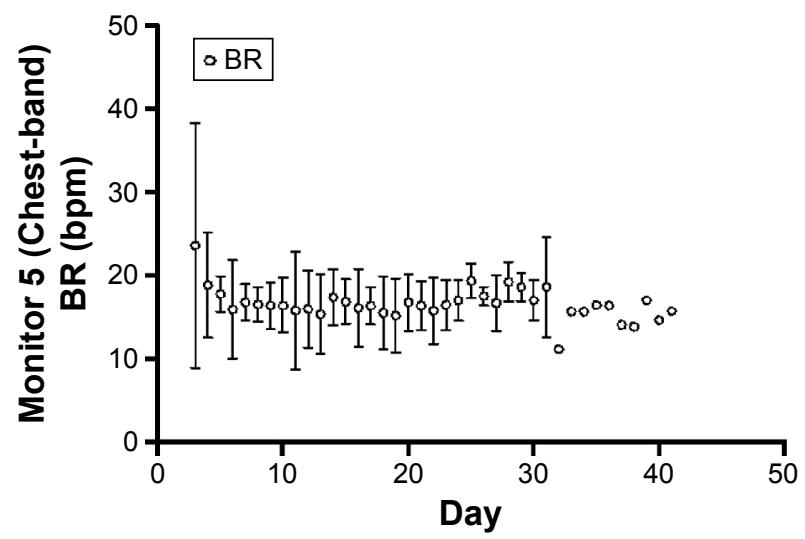

Figure 3 Evolution of BR shows the overall patient evolution of the BR during the recovery.

Abbreviation: $\mathrm{BR}$, breathing rate.

exacerbation (Figures 3 and see the file online supplement in $\underline{\mathrm{S} 3}$ ). However, this average result masks marked individual variation between patients, examples of which are shown in Figures 4 and see the file online supplement in S4. Additionally, there is a risk that as withdrawals were not included in the later time points and any observed trend in the mean could be misleading, especially if those dropping out did so because they felt too ill to continue. As was expected, some did not show changes in BR throughout the assessment period (eg, patient 18). The Supplementary material (see the file online supplement) provides the graphs for all participants recording data for at least 5 days.

Interestingly, one patient showed an increase of symptoms while being monitored, which resulted in hospital admission. The recovery in BR and HR was interrupted and an increase in these parameters was seen in the days prior to hospitalization (Figure 5).

\section{Discussion Main findings}

We established the accuracy of two novel BR monitors compared with the current gold standard, the acceptability to the patients of wearing the devices at home and the degree to which resting $\mathrm{BR}$ varied in the home setting. Of the two monitors that we found were valid tools to monitor BR, one captured data more reliably and was preferred by patients in terms of acceptability and was, therefore, selected for phase III where we aimed to detect a change in BR as people recovered from COPD exacerbations.

In phase III, we were able to demonstrate a mean gradual decrease in resting BR over time following an exacerbation in the population studied. However, individual data showed 

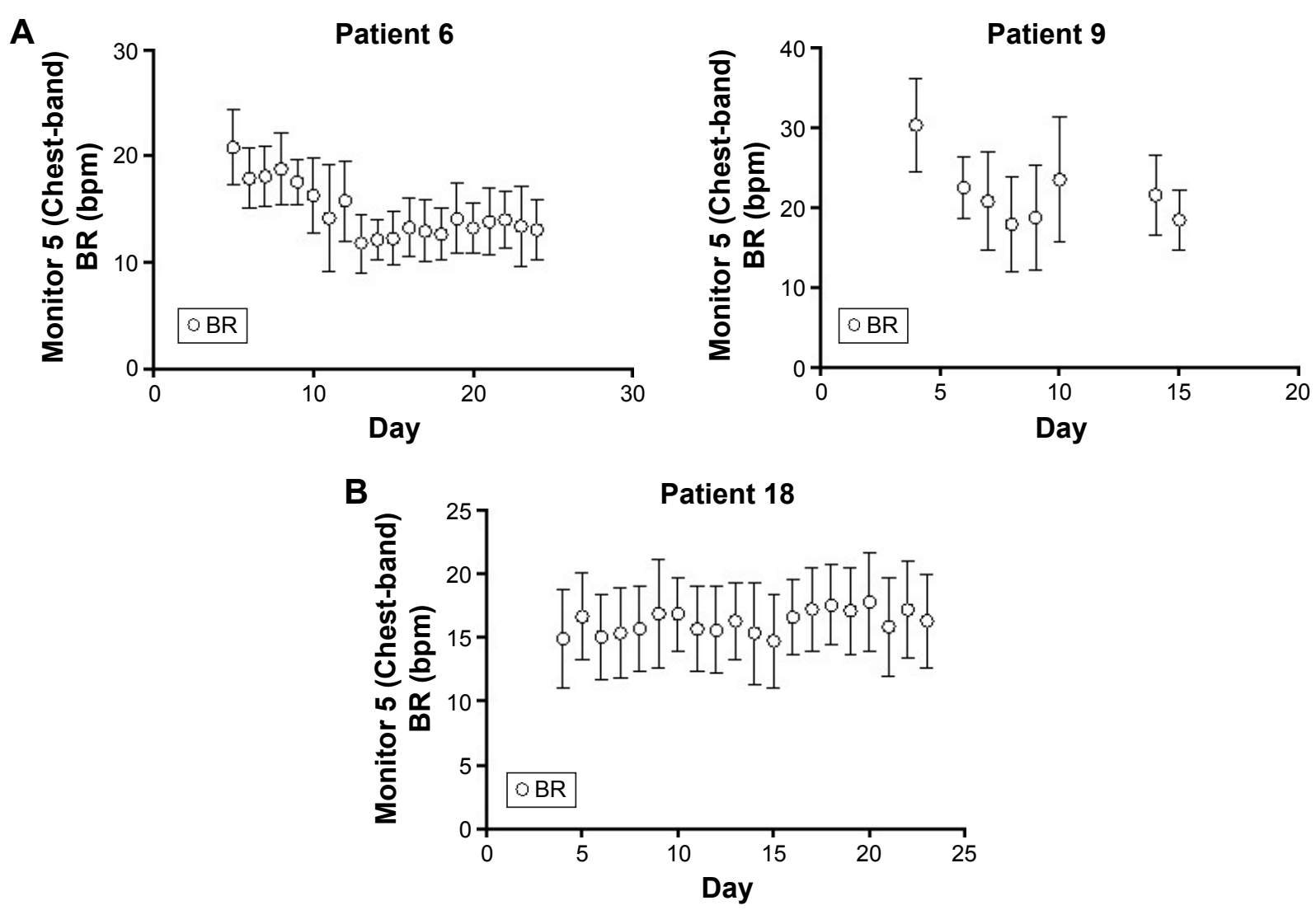

Figure 4 Example of patients during the monitoring period.

Notes: Panel (A) shows patients 6 and 9 who showed improvements during the recovery process in BR and Panel (B) is an example of a patient who did not show improvements.

Abbreviations: bpm, breath per minute; BR, breathing rate.

variation between patients with only a few patients showing the expected pattern of a decrease in BR, while for others BR remained stable throughout the whole period of assessment. In the one patient who was re-hospitalized due to a return of symptoms, the monitor captured an increase in BR (and also HR) in the days prior to hospitalization confirming that, when changes occur, the monitor was capable of capturing the information.

\section{Interpretation of findings in relation to previously published work}

To our knowledge this is the first evaluation of novel BR monitors in COPD against a gold standard in a range of people with COPD severity, in their homes. Research exploring the potential of using BR to predict exacerbations was published during the course of our study. ${ }^{27}$ The researchers
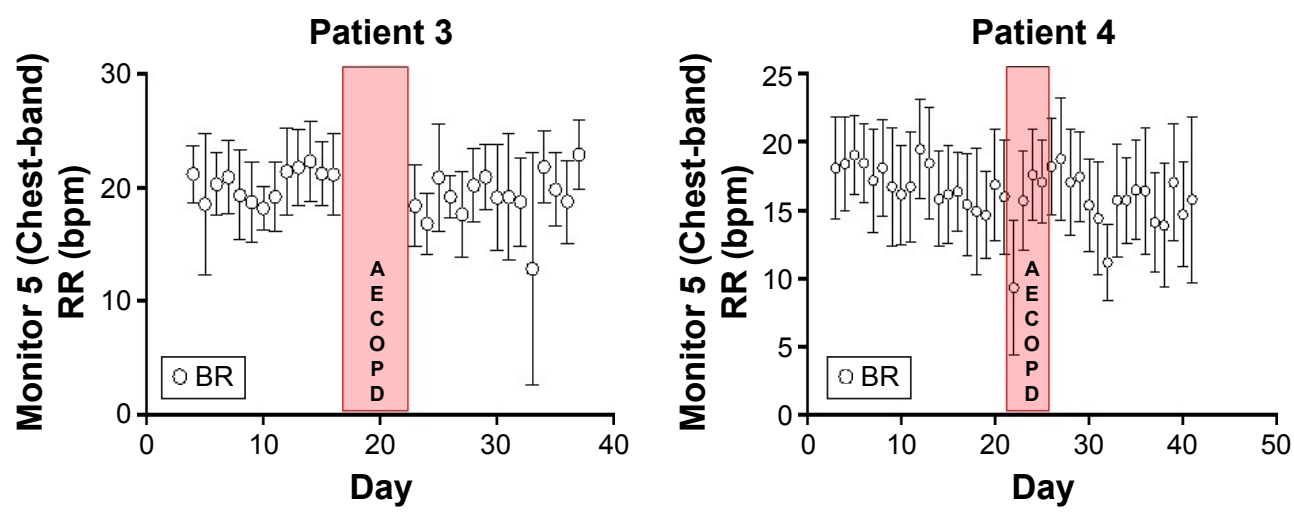

Figure 5 Monitored patients 3 and 4 during two cycles of AECOPD.

Abbreviations: bpm, breath per minute; $B R$, breathing rate; $A E C O P D$, exacerbations of chronic obstructive pulmonary disease; RR, respiratory rate. 
found that in people on domiciliary oxygen background variation was sufficiently low to allow prediction of an exacerbation. However, this cohort was probably more sedentary than our group, facilitating the measurement of resting BR.

Neither of the monitors in phase II provided immediate feedback on BR to the patients, which may have limited the potential benefits for the patient. The provision of a numerical reading of oxygen saturation is strongly valued by patients, who used it to explore their condition, reassure themselves about their status, inform their self-management, and improve access to professional help. ${ }^{28-30}$ In the absence of feedback, the BR did not assist self-management and would have required professional supervision with the associated risk of creating dependence. ${ }^{30}$ Future integration of these devices into a telehealth system will need to address how the readings can be used to support selfmanagement. ${ }^{28}$

\section{Strengths and limitations of this study}

We tested all devices against a recognized gold standard and in phase III were able to take advantage of the fact that the device we selected could measure energy expenditure to establish resting periods clearly. BR changes dramatically with different activities, and we only analyzed resting BR data. Considerable within-patient variances of resting BR were observed, which may have been due to patients reexacerbating during their recovery period.

Our study included people with a range of severities of COPD so that we could establish the utility of devices in measuring BR across the spectrum of disease. In contrast to phases I and II (in which participants were required to be stable), recruitment of patients early after the onset of AECOPD for phase III was difficult because of the patients' perception that they were too ill to take part in research. Consequently, we believe that some of the patients had returned to their baseline levels of BR at the time of recruitment, which may have contributed to the observation that measurements (BR, but also HR and $\mathrm{PAL}$ ) remained stable in these subjects during the assessment period.

\section{Implications for future research, policy, and practice}

We have demonstrated that accurately monitoring resting BR in ambulatory people with COPD is possible and potentially acceptable. The level of compliance with the use of the monitors was good particularly among the stable patients who participated in phase II who indicated that they would be willing to use either sensor for longer periods of time. There were some practical concerns related to acceptability (eg, skin reactions to adhesives, lights visible through clothing), which could be addressed as part of future development to customize these monitors for routine domiciliary use, and it is this relatively well group (as opposed to those recruited in phase 3 ) for whom a potential intervention would be targeted. Our study revealed ways in which acceptability might be improved (eg, by providing real-time patient feedback).

We undertook this study to explore the potential of BR monitoring in people with COPD with the ultimate aim of using such monitoring to improve the timely detection and self-management of exacerbations to prevent hospital admissions. The substantial background variation presented challenges in interpreting the signals of an exacerbation, and future development will need to address the detection of small changes in the context of background noise.

Monitoring recovery from an AECOPD was a preliminary step before embarking on a prospective study to determine if such a change was likely to be detectable in the other direction (as people at high risk of exacerbation fell ill with AECOPD). AECOPDs are heterogeneous and an appropriate approach may be in identifying the sub-group of patients in whom change in BR is detectable and predictive of exacerbations. ${ }^{1}$ Changes in breathlessness, ${ }^{31} \mathrm{BR},{ }^{27,32}$ and $\mathrm{HR}^{33}$ increase while PALs $^{34}$ decrease during AECOPD. Our study proved the validity of a commercially available monitor to capture these parameters, which can help the early identification of patients at risk of exacerbations and future hospitalizations in order to improve patients' self-management and management by health care professionals.

\section{Conclusions}

Accurately monitoring of resting BR is possible in ambulant people with COPD. Compliance and acceptability was high among those patients who were stable but was challenging for some who were very ill, although immediate feedback on BR to patients might have improved acceptability. A mean fall in resting BR during the recovery phase of an AECOPD masked substantial heterogeneity at an individual patient level and it was not clear that such changes could reliably be identified from normal background variation. A prospective study among those at risk of an exacerbation is needed to assess changes in BR at the onset of AECOPD.

\section{Acknowledgments}

We thank Lucy McCloughan for her invaluable help in the management, coordination, and reporting of this study. 
We thank all the patients who took part, the Community Respiratory Team in Edinburgh and the staff of the Respiratory Unit at the Royal Infirmary of Edinburgh for their invaluable help in recruiting patients during phase III of this study. We also thank Abel Rubio Fornes for help in the data management.

The study was funded by the Chief Scientist Office of the Scottish Government CZH/4/826. CJW, RAP, JH, and BM were supported in this work by NHS Lothian via the Edinburgh Health Services Research Unit. LCM is partly funded by an Engineering and Physical Sciences Research council (EPSRC)/Philips Healthcare grant and the College of Medicine and Veterinary Medicine of The University of Edinburgh.

\section{Author contributions}

All authors contributed toward data analysis, drafting and revising the paper and agree to be accountable for all aspects of the work.

\section{Disclosure}

The authors report no conflicts of interest in this work.

\section{References}

1. Mathers CD, Loncar D. Projections of Global Mortality and Burden of Disease from 2002 to 2030. PLoS Med. 2006;3(11):e442.

2. Wilkinson TM, Donaldson GC, Hurst JR, Seemungal TA, Wedzicha JA. Early therapy improves outcomes of exacerbations of chronic obstructive pulmonary disease. Am J Respir Crit Care Med. 2004;169(12): 1298-1303.

3. Bourbeau J. Preventing hospitalization for COPD exacerbations. Semin Respir Crit Care Med. 2010;31(3):313-320.

4. Effing T, Kerstjens H, van der Valk P, Zielhuis G, van der Palen J. (Cost)-effectiveness of self-treatment of exacerbations on the severity of exacerbations in patients with COPD: the COPE II study. Thorax. 2009;64(11):956-962.

5. Bischoff EW, Hamd DH, Sedeno M, et al. Effects of written action plan adherence on COPD exacerbation recovery. Thorax. 2011; 66(1):26-31.

6. Rice KL, Dewan N, Bloomfield HE, et al. Disease management program for chronic obstructive pulmonary disease: a randomized controlled trial. Am J Respir Crit Care Med. 2010;182(7):890-896.

7. Effing T, Monninkhof EM, van der Valk PD, et al. Self-management education for patients with chronic obstructive pulmonary disease. Cochrane Database Syst Rev. 2007;17(4):CD002990.

8. Trappenburg JC, Monninkhof EM, Bourbeau J, et al. Effect of an action plan with ongoing support by a case manager on exacerbation-related outcome in patients with COPD: a multicentre randomised controlled trial. Thorax. 2011;66(11):977-984.

9. Hubbard R. The Burden of Lung Disease. Thorax. 2006;61(7): $557-558$.

10. The Commission To The European Parliament, The Council, The European Economic And Social Committee And The Committee Of The Regions. eHealth Action Plan 2012-2020 - Innovative healthcare for the 21st century. Brussels, Belgium; 2012. Available from: http:// ec.europa.eu/health/ehealth/docs/com_2012_736_en.pdf. Accessed July 30, 2016.
11. NHS-Scotland, Long Term conditions Collaborative. Improving Self Management Support; 2009. Available from: http://www.gov.scot/ resource/doc/274194/0082012.pdf. Accessed July 30, 2016.

12. Daniel H, Sulmasy LS; Health and Public Policy Committee of the American College of Physicians. Policy recommendations to guide the use of telemedicine in primary care settings: an American College of Physicians position paper. Ann Intern Med. 2015;163(10):787-789.

13. Steventon A, Bardsley M, Billings J, et al. Effect of telehealth on use of secondary care and mortality: findings from the Whole Systems Demonstrator cluster randomised trial. Age Ageing. 2013;42(4): 501-508.

14. Pinnock H, Hanley J, McCloughan L, et al. Effectiveness of telemonitoring integrated into existing clinical services on hospital admission for exacerbation of chronic obstructive pulmonary disease: researcher blind, multicentre, randomised controlled trial. $B M J$. 2013;347:f6070.

15. van Dyk L. A review of telehealth service implementation frameworks. Int J Environ Res Public Health. 2014;11(2):1279-1298.

16. Burton C, Pinnock H, McKinstry B. Changes in physiological measures with symptoms and decision to treat exacerbations of chronic obstructive pulmonary disease: analysis of telemonitoring data. $J$ Telemed Telecare. 2015;21:29-36.

17. Franciosi LG, Page CP, Celli BR, et al. Markers of exacerbation severity in chronic obstructive pulmonary disease. Respir Res. 2006;7:74.

18. Decramer MA, Borbeau J, Celli B, et al. Global Initiative for chronic obstructive pulmonary disease. Global strategy for the diagnosis, management, and prevention of chronic obstructive pulmonary disease; 2015. Available from: http:/www.goldcopd.org/uploads/users/files/ GOLD_Report_2015.pdf. Accessed July 30, 2016.

19. Miller MR, Hankinson J, Brusasco V, et al. Standardisation of spirometry. Eur Respir J. 2005;26(2):319-338.

20. Spruit MA, Gosselink R, Troosters T, et al. Muscle force during an acute exacerbation in hospitalised patients with COPD and its relationship with CXCL8 and IGF-I. Thorax. 2003;58(9):752-756.

21. Nyberg A, Saey D, Maltais F. Why and How limb muscle mass and function should be measured in patients with chronic obstructive pulmonary disease. Ann Am Thorac Soc. 2015;12(9):1269-1277.

22. McArdle WD, Katch FI, Katch VL, editors. Essentials of Exercise Physiology: Energy Expenditure During Rest and Physical Activity, 3rd ed. Philadelphia, PA: Lippincott Williams \& Wilkins; 2005:262-288.

23. Bland JM, Altman DG. Measuring agreement in method comparison studies. Stat Methods Med Res. 1999;8(2):135-160.

24. Corbin J, Strauss A. Basics of Qualitative Research: Techniques and Procedures for Developing Grounded Theory 3rd ed. Los Angeles, CA: Sage Publications Inc.; 2008.

25. R Core Team 2015. R: A Language and Environment for Statistical Computing. R Foundation for Statistical Computing, Vienna, Austria. Available from: https://www.R-project.org/. Accessed July 30, 2016.

26. IBM Corp. IBM SPSS Statistics for Windows, Version 21.0. Armonk, NY: IBM Corp; 2012.

27. Yañez AM, Guerrero D, Pérez de Alejo R, et al. Monitoring breathing rate at home allows early identification of COPD exacerbations. Chest. 2012;142(6):1524-1529.

28. MacNab M, Lee SH, McCloughan L, Hanley J, McKinstry B, Pinnock H. Oximetry-supported self-management for chronic obstructive pulmonary disease: mixed method evaluation of a pilot project. BMC Health Serv Res. 2015;15:485.

29. Ure J, Pinnock H, Hanley J, et al. Piloting tele-monitoring in Chronic Obstructive Pulmonary Disease: a mixed methods exploration of issues in design and implementation. Prim Care Respir J. 2012;21(1):57-64.

30. Fairbrother P, Pinnock H, Hanley J, et al. Exploring telemonitoring and self-management by patients with chronic obstructive pulmonary disease: a qualitative study embedded in a randomized controlled trial. Patient Educ Couns. 2013;93(2):403-410.

31. Williams V, Hardinge M, Ryan S, Farmer A. Patients' experience of identifying and managing exacerbations in COPD: a qualitative study. NPJ Prim Care Respir Med. 2014;24:14062. 
32. Stevenson NJ, Walker PP, Costello RW, Calverley PM. Lung mechanics and dyspnea during exacerbations of chronic obstructive pulmonary disease. Am J Respir Crit Care Med. 2005;172(12):1510-1516.

33. Stiell IG, Clement CM, Aaron SD, et al. Clinical characteristics associated with adverse events in patients with exacerbation of chronic obstructive pulmonary disease: a prospective cohort study. CMAJ. 2014;186(6):E193-E204.
34. Pitta F, Troosters T, Probst VS, Spruit MA, Decramer M, Gosselink R. Physical activity and hospitalization for exacerbation of COPD. Chest. 2006;129(3):536-544.

International Journal of COPD

\section{Publish your work in this journal}

The International Journal of COPD is an international, peer-reviewed journal of therapeutics and pharmacology focusing on concise rapid reporting of clinical studies and reviews in COPD. Special focus is given to the pathophysiological processes underlying the disease, intervention programs, patient focused education, and self management protocols

\section{Dovepress}

This journal is indexed on PubMed Central, MedLine and CAS. The manuscript management system is completely online and includes a very quick and fair peer-review system, which is all easy to use. Visit http://www.dovepress.com/testimonials.php to read real quotes from published authors.

Submit your manuscript here: http://www.dovepress.com/international-journal-of-chronic-obstructive-pulmonary-disease-journal 